\title{
Traumatic Pseudolipoma of the Buccal Fat Pad
}

Kenji Takasaki, M. D. ${ }^{1)}$, Chiharu Kihara, M. D. ${ }^{1)}$

Kaori Enatsu, M. D. ${ }^{1)}$, Hidetaka Kumagami, M. D. ${ }^{1)}$

Haruo Takahashi, M. D. ${ }^{1)}$

1) Department of Otolaryngology, Head and Neck Surgery,

Nagasaki University Graduate School of Biomedical Sciences

Address:

Department of Otolaryngology, Head and Neck Surgery,

Nagasaki University Graduate School of Biomedical Sciences

1-7-1, Sakamoto, Nagasaki 852-8501, Japan

Correspondence / Reprint requests:

Kenji Takasaki, M. D.

Department of Otolaryngology, Head and Neck Surgery,

Nagasaki University Graduate School of Biomedical Sciences

1-7-1, Sakamoto, Nagasaki 852-8501, Japan

Phone: +81-95-849-7349, Fax: +81-95-849-7352

E-mail:ktakasa@nagasaki-u.ac.jp

The parents of this patient were informed and consented to report the present paper. 


\section{Introduction}

Traumatic pseudolipoma of the buccal fat pad (intraoral herniation of the

buccal fat pad) is a rare traumatic disease among young children. It typically presents

as an expanding pedunculated mass of soft tissue arising from the buccal mucosa

following a minor trauma to the buccal soft tissues. We report a case with traumatic

pseudolipoma of the buccal fat pad in a young girl, and review pertinent literature.

\section{Case Report}

A 26-month-old Japanese girl visited our outpatient clinic presenting a

growing large intraoral mass. Her grandmother stated that the girl had been playing in the house with a toothbrush in her mouth. She was found lying on the floor with a bleeding oral wound. Her mother stated that a mass appeared from the wound and rapidly increased in size in one day. It was noted that a large, irregular, reddish mass arose from the left buccal mucosa (Figure 1). She had a history of asthma treated by inhalation, but was otherwise normal. Her white blood cell count was 23,500 with 68\% segmented neutrophils, and her C-reactive protein was $3.58 \mathrm{mg} / \mathrm{dl}$. Other laboratory data was essentially normal. Computed tomography (CT) revealed that her 
left buccinator muscle was damaged with cellulites, and that a part of her buccal fat pad had prolapsed into her oral cavity (Figure 2).

Under general anesthesia, the protruded mass was excised with minimal bleeding and the wound was left open. The base of the mass arose from the left buccal mucosa approximately one centimeter posteroinferior to the orifice of the Stenson's duct, and deeply attached to the buccal fat pad. The postoperative course was uneventful and she was discharged five days after the operation. No sequelae including facial deformity was recognized at the follow-up examination one month after surgery.

Histopathological examination of the excised mass revealed an adipose tissue separated by connective tissue stroma composed of collagen fibers and blood vessels. Inflammatory cell infiltration was also present. The overall histopathologic features were compatible with traumatic pseudolipoma of the buccal fat pad.

\section{Discussion}

Pseudolipoma of the buccal fat pad (traumatic herniation of the buccal fat pad) into the oral cavity was first reported in literature by Clawson in $1968{ }^{1}$. Until now, approximately 30 cases reported have described traumatic pseudolipoma of the 
buccal fat pad in literature ${ }^{1,2}$. This disease event typically occurs in children ranging from four months to four years of age when the fat pad is still quite large in proportion to the size of the face. Stuzin et al. ${ }^{3}$ describes the buccal fat pad as a rounded, biconvex structure with a thin but distinctive capsule lying between the buccinator and masseter muscles. It consists of a main body with four main extensions: buccal, pterygoid, superficial, and deep temporal. The buccal fat pad is located within the masticatory space, and is in intimate association with the muscles of mastication, the parotid duct, and the facial nerve. It serves as a lining of the masticator space, and is believed to aid in facilitating muscular movement.

The etiology of traumatic pseudolipoma of the buccal fat pad is speculated as follows: Infants and young children have frequent chances of falling down with an instrument such as a toothbrush in their mouth, and getting an oral cavity injury. Furthermore, negative intra oral pressure induced by their suckling activity may promote herniation of the buccal fat pad from the wound. Therefore we should observe their clinical course carefully, even if the wound in buccal mucosa is small, particularly in cases of infants and children. 
The differential diagnoses include pyogenic granuloma, inflammatory

pseudotumor, foreign body granuloma, traumatic neuroma, lipoma, hemangioma, and salivary neoplasm. Generally, a history of trauma, an absence of mass before the accident, the specific anatomic site, and adipose appearance serves to diagnosis of traumatic herniation of the buccal fat pad.

Few clinical image reports exist about this disease. At present, the CT demonstrates that the left buccinator muscle was damaged with cellulites, and that most part of the buccal fat pad prolapsed into the oral cavity. The present CT findings were of great help to us for diagnoses. Furthermore, magnetic resonance imaging may have been even more effective in diagnosis of this disease. The present case is the first clinical report of this disease via imaging study.

Histopathological examination revealed a connective tissue stroma with sheets and groups of adipocytes without atypia and no epithelial cover. The interstitial spaces are often occupied with extravasated red cells and acute or chronic inflammatory infiltrates. Focal thrombosis and necrosis have also been described. 
procedures. When discovered in the early stages, the protruded mass is small with minimal inflammatory changes and the lesion can be repositioned immediately. If the mass is too large to replace in the limited laceration injury site or necrosis already takes place in the mass, it is recommended that one excise the mass at the base. With either method, care should be taken to avoid injury to the Stenson's duct and orifice. 


\section{References}

1. Clawson JR, Kline KK, Armbrecht EC. Trauma-induced avulsion of the buccal fat pad into the mouth Report of case. J Oral Surg 1968;26:546-547.

2. Desai RS, Vanaki SS, Puranik RS, et al. Traumatic herniation of buccal fat pad (traumatic pseudolipoma) in a 4-year-old boy: a case report. J Oral Maxillofac Surg. 2005;63:1033-1034.

3. Stuzin JM, Wagstrom L, Kawamoto HK, et al. The anatomy and clinical applications of the buccal fat pad. Plast Reconstr Surg 1990;85:29-37. 


\section{Legend}

Figure 1.

Clinical appearance showed that the herniated mass $(*)$ protrudes from the left buccal mucosa (B). T: Tongue, S: Soft palate

Figure 2.

Computed tomography revealed that left buccinator muscle (white small arrows) was damaged (arrowhead) with cellulites. Buccal fat pad (large arrow) was located in her oral cavity. 


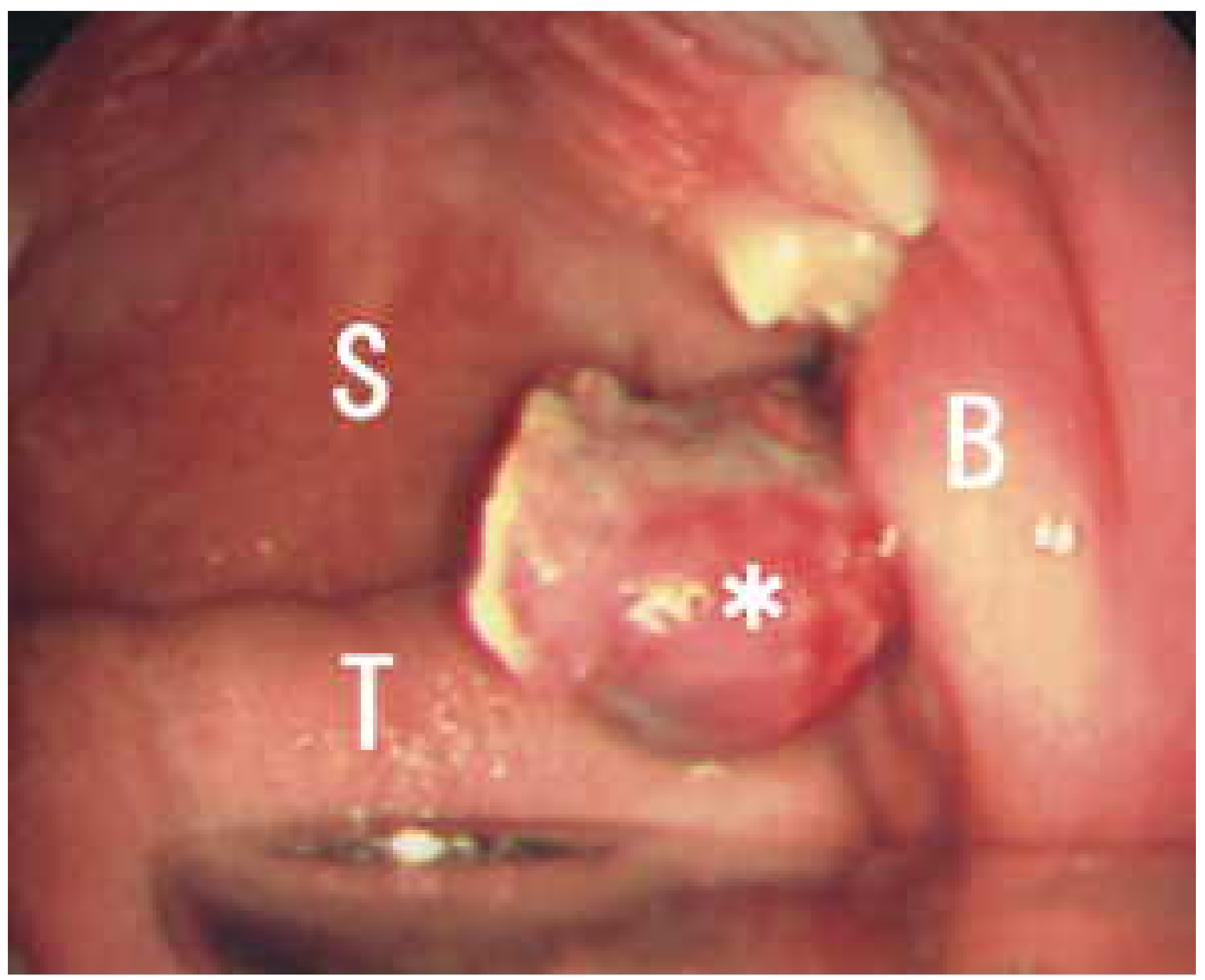




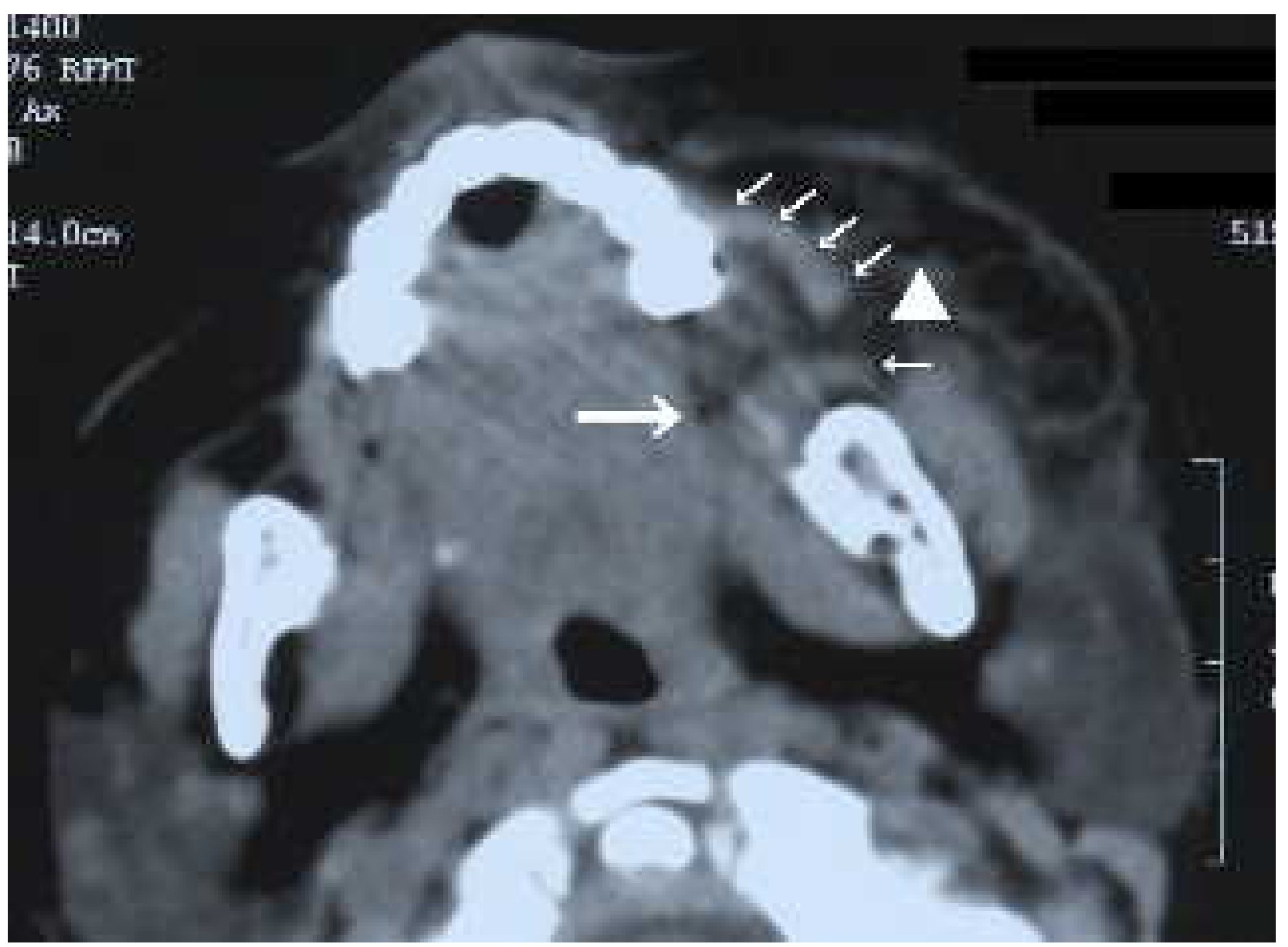

